\title{
EVALUATION OF NEEDLE BIOPSY OF SYNOVIAL MEMBRANE
}

\author{
BY \\ MATTHEW WILKINSON AND BRIAN S. JONES \\ From the Clinical Research Department, Charing Cross Hospital, and the Department of Pathology, \\ St. Bartholomew's Hospital, London
}

Although first described in 1951 (Polley and Bickel, 1951), needle biopsy of synovial membrane has not come into such common usage as that of liver, pleura, or even kidney. Judging from the few American reports dealing with its diagnostic value (Mikkelsen, Duff, Castor, Zevely, and French, 1958; Polley, Bickell and Dockerty, 1954; Rodnan, Yunis, and Totten, 1960; Schwartz and Cooper, 1961), the main disadvantages seem to be:

(1) Owing to the variability of synovial histology within the same joint, particularly in disease (Cruickshank, 1952), the small specimen obtained by blind biopsy may not be representative or may completely miss any specific change, particularly if this is focal as in tuberculosis or gout.

(2) Only large joints (mainly knees) are suitable for this procedure.

(3) In most arthropathies a diagnosis can be made from the other clinical features, if not on first examination, then after a period of observation, and a biopsy is seldom needed.

(4) Histological changes in the synovial membrane are rarely specific and may only indicate a pattern common to several arthropathies, e.g. rheumatoid arthritis, ankylosing spondylitis, and Reiter's syndrome.

On the other hand, needle biopsy is a simple out-patient procedure with no more risk and causing no more discomfort than a simple aspiration of synovial fluid. For this reason we have taken a needle biopsy whenever a joint has required aspiration during the past 2 years. In the majority of cases the diagnosis was not in doubt and only occasionally did the biopsy contribute to the final diagnosis.

\section{Methods}

Biopsies were taken with a Harefield pleural biopsy needle (Abrams, 1958) sharpened to a point with an 8-mm. bevel suitable for penetrating the less rigid periarticular tissues. This needle, which is similar to the Polley-Bickel needle, allows aspiration, injection, and biopsy with only one penetration.

With large effusions the needle was inserted into the medial aspect of the suprapatellar pouch just above the patella, but with small effusions the medial infrapatellar space was easier to enter. Local anaesthetic was liberally infiltıated down to the synovial space and the needle inserted through a small skin incision. After the needle was opened, synovial fluid escaped under gentle external pressure. For the biopsy, the needle was swung round so that it entered the joint obliquely with the side opening lying alongside the synovial membrane. Pressure applied to the skin over the end of the needle caused a nipple of synovial membrane to enter the side opening of the needle and this could be snipped off by rotating the inner cutting needle.

Sections from all biopsies were stained with haematoxylin and eosin, and further sections from many biopsies were stained with Unna-Pappenheim's stain to show up plasma cells.

\section{Results}

86 biopsies were attempted on 74 patients, but in seventeen instances ( 23 per cent.) the specimen obtained was inadequate or contained no synovial tissue. This was usually due to fibrinous exudate blocking the needle or occasionally to poor positioning of the needle so that the biopsy was taken from periarticular tissue. Although the success rate improved with practice, approximately 10 per cent. of even the more recent attempts produced an unsatisfactory specimen. It proved particularly difficult to take a specimen from large knee joints with thick periarticular tissues by this method.

The only complication encountered was moderate subcutaneous bruising in one case.

Further comments will apply only to the 69 successful biopsies taken from sixty patients. 
Rheumatoid Arthritis. - 34 satisfactory specimens were obtained from 28 patients. The latter were classified according to the revised criteria of the American Rheumatism Association (Ropes, Bennett, Cobb, Jacox, and Jessar, 1959) but without consideration of the biopsy results, as classical (13 cases), definite (13 cases), and probable ( 2 cases). In all except one case, synovial fluid was available and the values for total protein exceeded $4 \mathrm{~g}$. per cent. and those for gamma globulin exceeded $1 \mathrm{~g}$. per cent. These changes have been found to be almost invariable in rheumatoid arthritis (Wilkinson and Jones, 1962). In none of these cases did the histological interpretation conflict with the clinical diagnosis. The interpretation was usually easy and the commonly described histological features were seen, viz. fibrinous or fibrinopurulent exudate, hyperplasia of the synoviocytes, increased vascularity, and diffuse or sometimes focal accumulations, especially in relation to blood vessels, of variable proportions of lymphocytes and plasma cells. Binucleate plasma cells were not unusual. Oedema and fibrosis, though sometimes seen, were more difficult to assess owing to distortion of the small specimens. In six cases biopsies were taken within

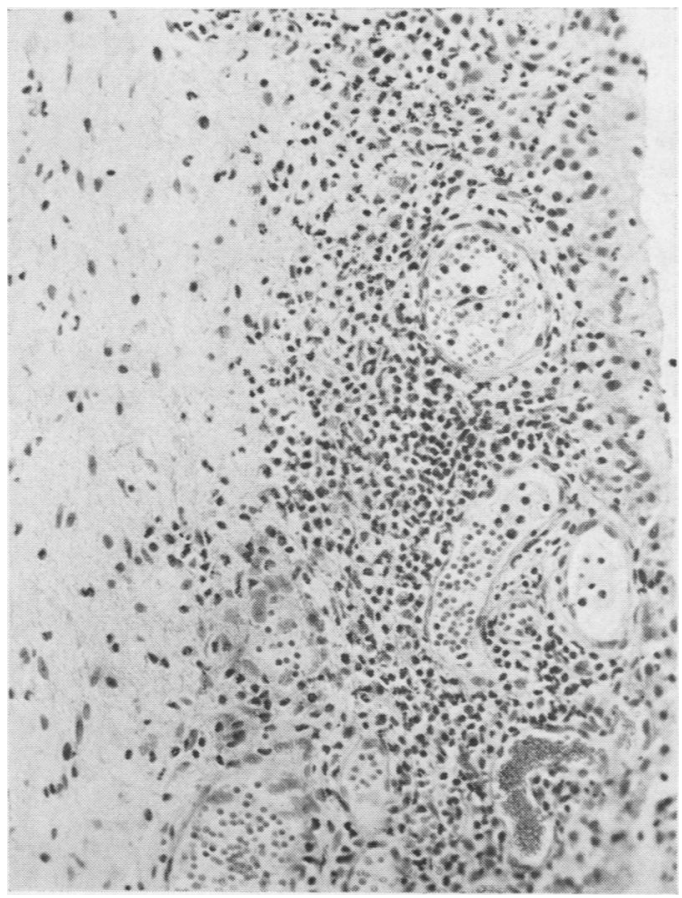

Fig. 1.-Synovium in rheumatoid arthritis one week after onset of joint swelling. Inflammatory changes localized to superficial zone. $\times 133$.
3 months of onset of the disease and usually within a much shorter interval of the onset of symptoms in the particular joint aspirated. In these the changes were strikingly confined to the synovial cell layer and the immediate subsynovial zone, and the infiltrating cells sometimes included a moderate number of neutrophils (Figs 1 and 2). Similar changes have been reported in early rheumatoid arthritis by Kulka, Bocking, Ropes, and Bauer (1955) and in early cases of Reiter's syndrome by Weinberger, Ropes, Kulka, and Bauer (1962). Although Kulka (1962) considered that the changes in early cases of Reiter's syndrome could be distinguished from those in rheumatoid arthritis of comparable duration by the intensity of diffuse hyperaemia, the even superficial distribution of the cellular exudate, and the relatively marked neutrophil infiltrate, we would find this a very difficult distinction to make judging from what we have seen in early cases of rheumatoid arthritis.

One biopsy from a patient with classical rheumatoid arthritis (23 years polyarthritis, extensive radiological erosions, and positive tests for rheumatoid factor) showed in addition to the usual histological changes an extensive infiltration with

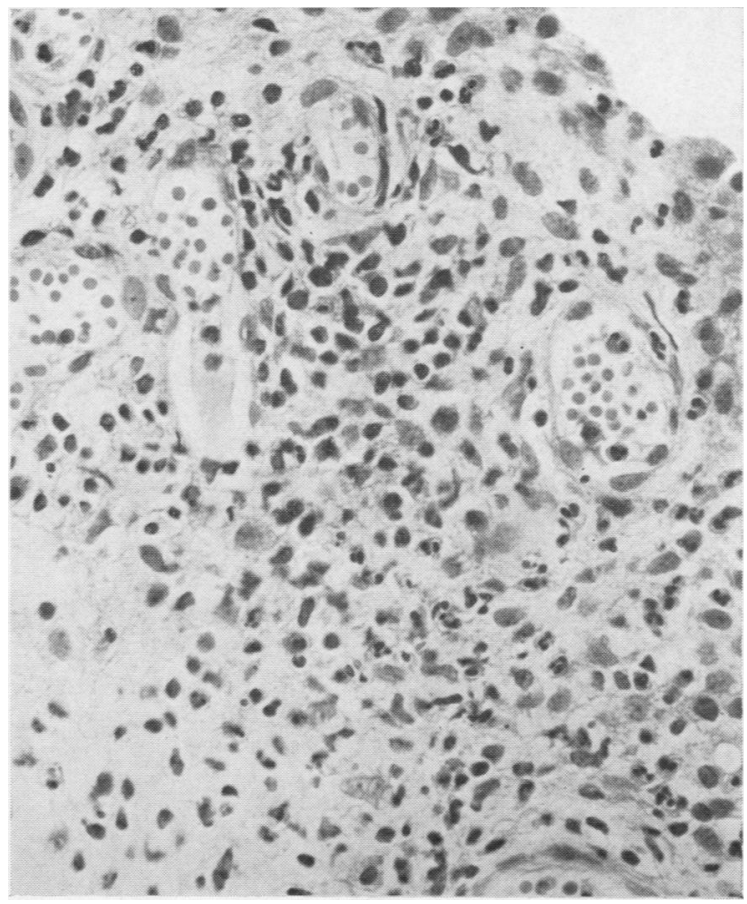

Fig. 2.-High-power view of superficial zone of synovium shown in Fig. 1 to demonstrate neutrophil infiltration. $\times 350$. 
macrophages containing inclusions of homogeneous eosinophilic material (Fig. 3) which also stained with Schiff's periodic acid stain. This does not appear to be a recognized feature of rheumatoid arthritis and for this reason we consider that the inclusions may have resulted from past intraarticular injections of a steroid preparation containing polyethylene glycol. This possibility is being investigated.

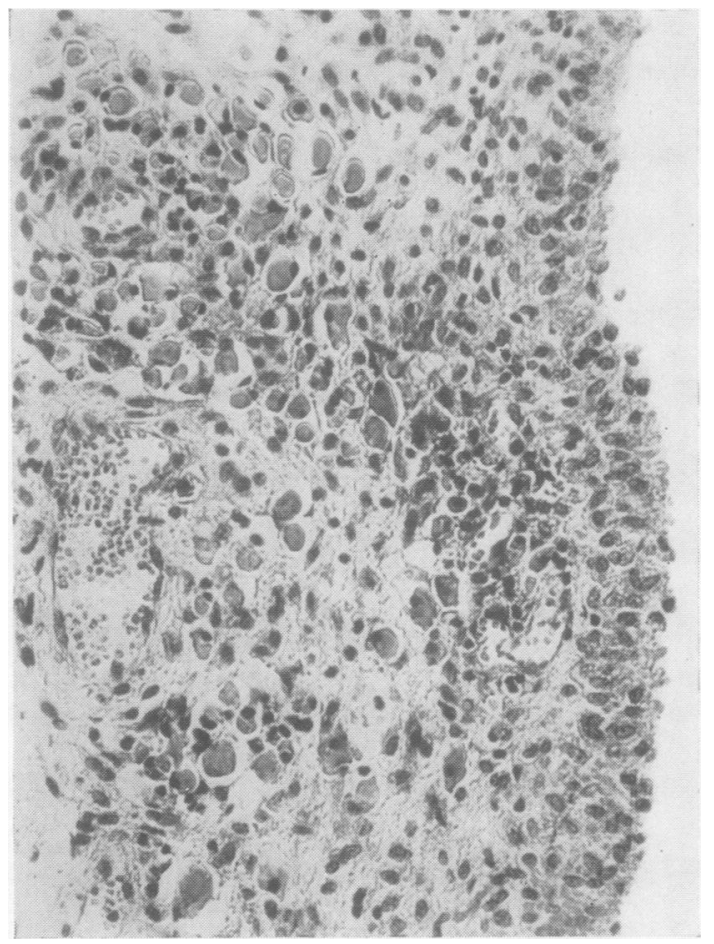

Fig. 3.-Eosinophilic inclusions within macrophages scattered throughout synovium of a rheumatoid joint recently injected with a steroid preparation. $\times 188$.

Ankylosing Spondylitis.-Three satisfactory biopsies taken from two patients showed histological changes indistinguishable from those seen in rheumatoid arthritis.

Osteo-Arthritis. - 25 successful biopsies were taken from 23 patients. All were middle-aged or elderly, often with a history of occupational or accidental trauma, a recent or chronic effusion into one or rarely both knees, and radiological changes compatible with osteo-arthritis. Tests for rheumatoid factor were available in most cases and were negative, and the erythrocyte sedimentation rate was usually normal, but occasionally mildly accelerated during the early stages of an effusion.
Synovial fluid protein values were below $4 \mathrm{~g}$. per cent. and the electrophoretic patterns suggested a non-rheurnatoid disorder (Wilkinson and Jones, 1962).

Until we became accustomed to the range of histological changes in osteo-arthritis, these specimens proved rather more difficult to interpret, for any of the features seen in rheumatoid arthritis may be seen in osteo-arthritis though usually in lesser degree. Fibrinous exudate, though not prominent, was seen in nine biopsies. The appearance of the synovial cell layer varied from almost normal to gross hyperplasia so that a layer several cells thick was present. The vascularity of the synovium likewise varied from normal to markedly increased. We were struck by the high proportion of biopsy specimens in osteo-arthritis which showed considerable hyperaemia. This would seem to be contrary to expectation in a chronic degenerative disease, and is probably related rather to the traumatic factors which caused, and in some cases maintained, the effusions. The same degree of hyperaemia may not necessarily be seen in osteoarthritis without joint effusions (Collins, 1949).

By far the most useful distinguishing feature was the paucity of the cellular infiltration, which in most cases consisted of lymphocytes, although in several cases unequivocal plasma cells were seen in moderate numbers (Fig. 4, opposite). Focal accumulations of lymphocytes were unusual and were seen in only two biopsies (Fig. 5, opposite), but these were solitary foci and no biopsy showed foci scattered throughout the sections as may be seen in rheumatoid arthritis. In three biopsies the cellular infiltrate was of moderate degree and these sections caused the greatest difficulty, although clinically there seemed no cause to question the diagnosis of osteo-arthritis.

One patient with long-standing osteo-arthritis also suffered from myelomatosis, but the histological picture of the synovium was one of uncomplicated osteo-arthritis.

Other Arthropathies.-Successful biopsies were taken from single cases of sarcoidosis, acute leukaemia, neuropathic arthropathy, and probable gout. In each one the histological picture showed no specific features that would distinguish it from the appearances seen in osteo-arthritis.

Biopsies were taken from three further patients in whom the diagnosis remains uncertain.

The first, a young man with an effusion into one knee joint for 2 months and synovial fluid protein changes suggestive of a rheumatoid disorder, has unfortunately been lost to follow-up. His biopsy showed no diagnostic features, with mild hyperplasia of the synoviocytes 


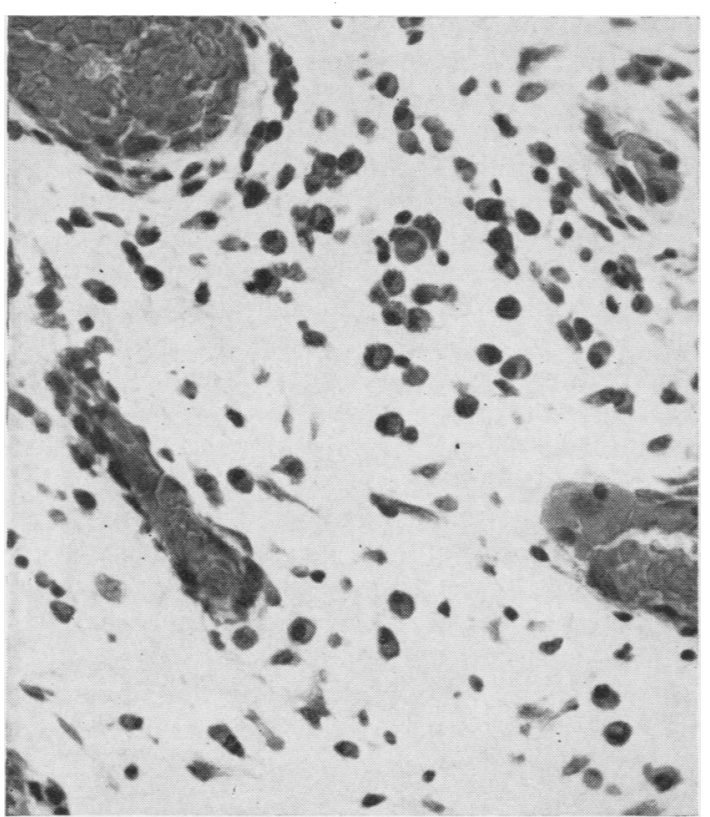

Fig. 4.-Collection of plasma cells in synovium of a patient with otherwise typical osteo-arthritis. $\times 385$.

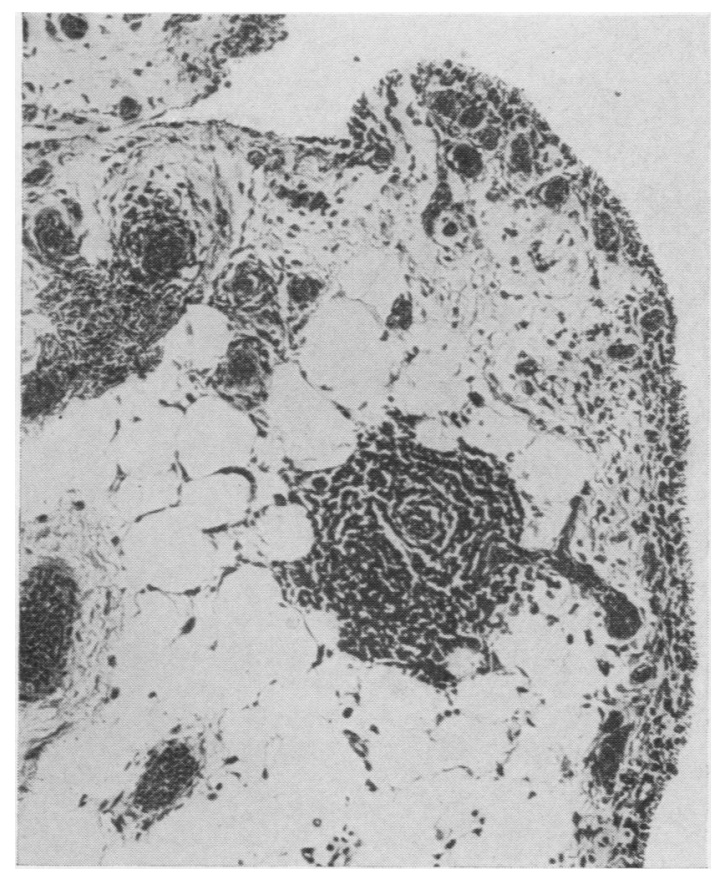

Fig. 5.-Lymphocytic focus in synovial membrane of an osteoarthritic joint. $\times 95$ and slightly increased vascularity, but virtually no abnormal cellular infiltration.

The second patient, a young woman on replacement therapy for hypopituitarism following operations for a pituitary tumour, gave a history of 3 years' polyarthritis. The erythrocyte sedimentation rate was accelerated, but the synovial biopsy showed no specific features and, although there was a mild infiltrate of lymphocytes, this was no more than we have seen in some cases of osteo-arthritis. The clinical diagnosis remains probable rheumatoid arthritis.

The third case illustrates the difficulty of interpreting some synovial biopsies from very early cases of probable rheumatoid arthritis. The patient was a woman of 28 years with a story of a painful swollen right knee for a period of 6 weeks some 5 years previously. Apart from occasional discomfort she then remained well and working until the present abrupt attack of pain and swelling of the right knee, associated with fever and accelerated sedimentation rate, but with normal radiographs and serum uric acid levels and with negative tests for rheumatoid factor. 10 days after the onset of the second attack, $80 \mathrm{ml}$. fluid were aspirated from the right knee and the total protein content $(5 \cdot 5 \mathrm{~g}$. per cent.) and electrophoretic studies revealed a rheumatoid pattern. At this time a needle biopsy (Fig. 6) showed a little firbinopurulent exudate, hyperplasia of the

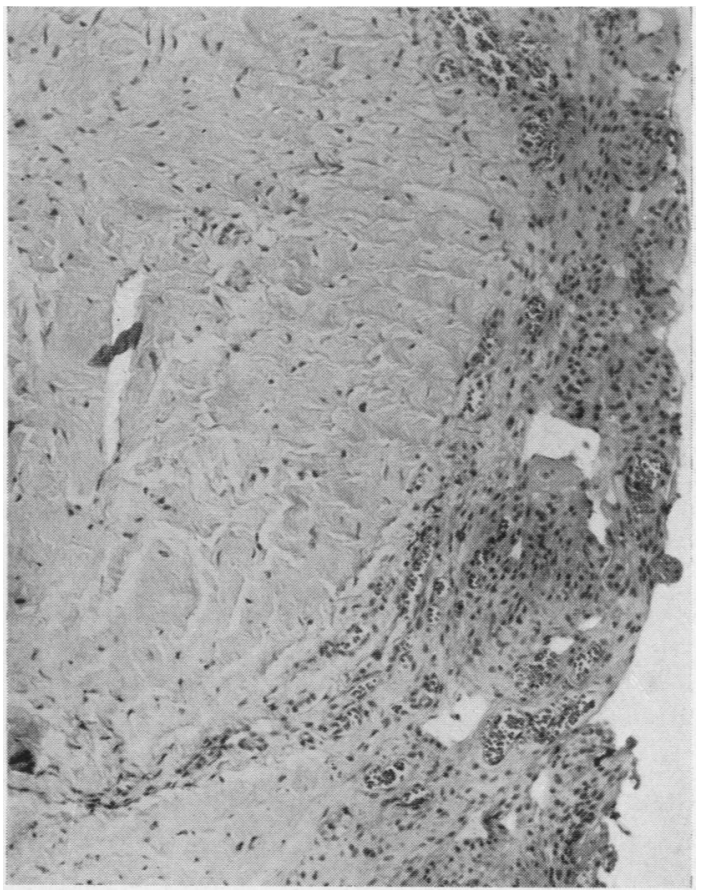

Fig. 6.-Synovial biopsy from probable rheumatoid arthritic 10 days after onset of effusion. There is hyperplasia of synoviocytes and mild hyperaemia, but only mild infiltration with lymphocytes and neutrophils. $\times 95$. 
synoviocytes, moderately increased vascularity especially of the subsynovial layer, and mild cellular infiltration with mostly lymphocytes, but also a scattering of neutrophils. It was impossible to give a definite opinion on this biopsy, so 24 days after the onset of swelling an arthrotomy was performed which revealed a thickened inflamed and haemorrhagic synovium and biopsies were taken from two different areas of synovium (Figs 7 and 8 ). The first showed a picture much more suggestive of rheumatoid arthritis, with a moderately dense cellular infiltration composed almost entirely of lymphocytes. However, the second biopsy illustrated quite strikingly the variability of the histological picture within the same joint, for it showed very little cellular infiltration and if considered alone would have led to a diagnosis of non-specific synovitis. It is easy to see how a needle biopsy taken from the region shown in Fig. 8 would be very misleading. Only 3 months after the onset of this second attack of arthritis the patient is well, apart from occasional twinges of pain in the right knee; nevertheless, the clinical diagnosis remains as probable rheumatoid arthritis.

\section{Discussion}

The simplicity and, if performed with full aseptic precautions, the safety of needle biopsy of synovial membrane, makes it well worthwhile in any case

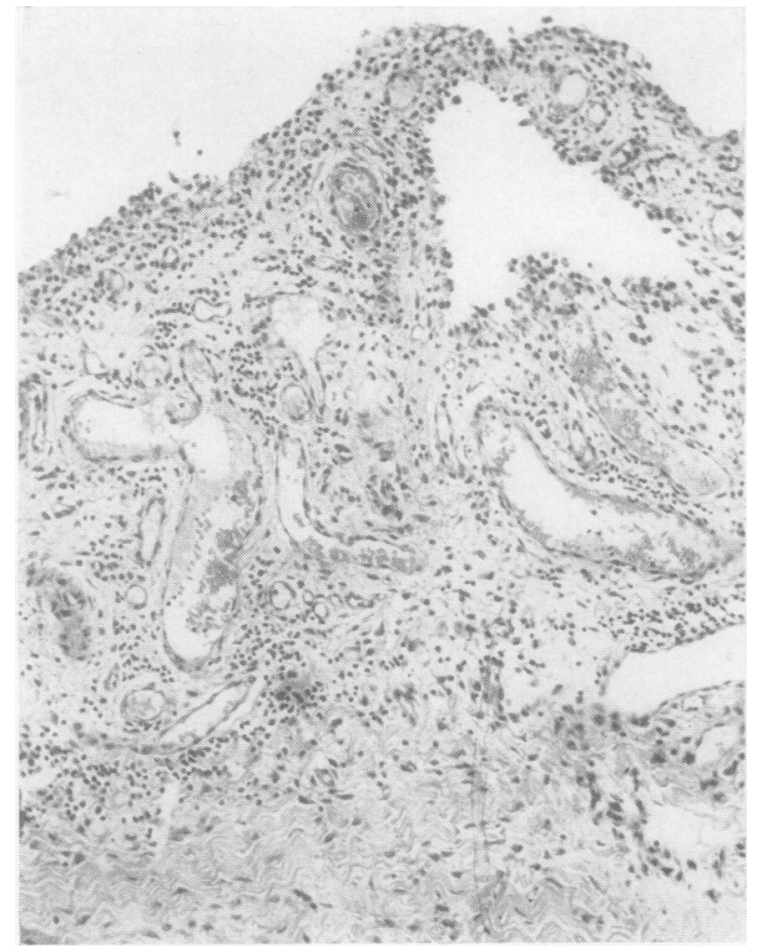

Fig. 7.-Biopsy from same joint as Fig. 6 taken 24 days after onset of effusion. This specimen shows more hyperaemia and a moderate infiltrate of lymphocytes. $\times 95$. in which synovial fluid is being aspirated for diagnostic reasons. Unfortunately the interpretation of the biopsy material is difficult, and it should be submitted to a pathologist with considerable experience of synovial pathology.

The biopsy report may merely supplement the clinical data, for the histological changes are rarely diagnostic of a specific disease, but the discovery of the granulomata of tuberculosis and sarcoidosis, or of the urate crystals in gout are pathognomonic. Not surprisingly, however, with such patchy lesions, the experience of other workers has shown that blind needle biopsy may miss the specific lesions, and the resulting picture may be at best nonspecific or at worst frankly misleading. For example, in cases of gout, synovial biopsies not showing urate crystals may show other changes resembling those seen in rheumatoid arthritis (Rodnan and others, 1960).

In most other diseases the synovial changes fall into one of two patterns: the rheumatoid pattern and the non-specific synovitis pattern.

The rheumatoid pattern is usually easily recognized, but is common to the arthropathies of rheu-

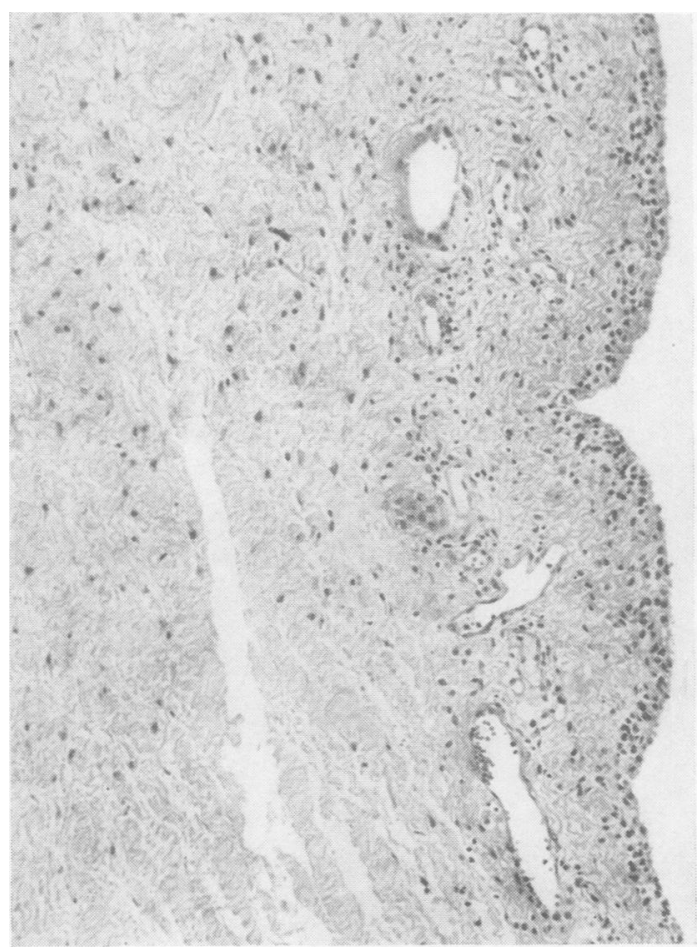

Fig. 8.-Simultaneous biopsy from a different part of the same joint as in Fig. 7, showing a variation of the histological picture. $\times 95$. 
matoid arthritis, Still's disease, ankylosing spondylitis, Reiter's syndrome, systemic lupus erythematosus, psoriasis, and Sjögren's syndrome (Mikkelsen and others, 1958). With early cases of rheumatoid arthritis and the other rheumatoid diseases, in which the cellular infiltration is less pronounced and includes fewer chronic and more acute examples of inflammatory cells, a confident histological diagnosis may be more difficult.

The non-specific synovitis pattern, though most commonly due to osteo-arthritis, is also seen in traumatic arthropathy such as may follow acute cartilage injury, and may also be seen in nonrheumatoid conditions such as gout where the changes already mentioned are not present. Furthermore, we believe that this non-specific pattern may occasionally be mimicked in the earliest stages of rheumatoid arthritis, when the inflammatory cell infiltration is sparse or patchy, as in Figs 6 and 8, and as described by Kulka and others (1955) in their Cases 2, 3, and 4.

In our experience the commonest mistake was an initial tendency to over-diagnose rheumatoid arthritis from the histological changes. This was because of our ignorance of the extent of the histological changes in osteo-arthritis with synovial effusion. These included marked synoviocyte hyperplasia and occasionally a surprising degree of chronic inflammatory cell infiltration, sometimes with modest numbers of plasma cells. In a few biopsies it may clearly be impossible to decide whether the changes represent an early rheumatoid change or a non-specific synovitis with more than usual cellular infiltration.

With these reservations in mind, we agree with Polley and Bickel (1951) that needle biopsy will prove to be of greatest value in cases of monoarthritis, since a clear-cut rheumatoid pattern of reaction, or the picture of one of the specific arthritic lesions may obviate a surgical biopsy. Unfortunately a needle biopsy showing the pattern of non-specific synovitis may mean very little and should not over-ride other clinical considerations or delay arthrotomy and surgical biopsy if this seems necessary.

\section{Summary}

The results of 69 successful needle biopsies from sixty arthritic knee joints have been analysed.
The value of the procedure and the difficulties of interpreting the histological material are discussed.

We are grateful to the Charing Cross Hospital Research Committee who made this study possible, to many physicians at Charing Cross, the Royal Northern, and St. Bartholomew's Hospitals for allowing us to study their patients, and to Mr. P. Crocker and Mr. K. W. Iles for the photomicrographs.

\section{REFERENCES}

Abrams, L. D. (1958). Lancet, 1, 30.

Collins, D. H. (1949). "The Pathology of Articular and Spinal Diseases", p. 97. Arnold, London.

Cruickshank, B. (1952). Ann. rheum. Dis., 11, 137.

Kulka, J. P. (1962). Arthr. and Rheum., 5, 195.

-, Bocking, D., Ropes, M. W., and Bauer, W. (1955) A.M.A. Arch. Path., 59, 129.

Mikkelsen, W. M. Duff, I. F., Castor, C. W., Zevely, H. A., and French, A. J. (1958). Arch. intern. Med., 102, 977.

Polley, H. F., and Bickel, W. H. (1951). Ann. rheum. Dis., 10, 277.

$\longrightarrow,-$, and Dockerty, M. B. (1954). Ibid., 13, 353.

Rodnan, G. P., Yunis, E. J., and Totten, R. S. (1960). Ann. intern. Med., 53, 319.

Ropes, M. W., Bennett, G. A., Cobb, S., Jacox, R., and Jessar, R. A. (1959). Ann. rheum. Dis., 18, 49.

Schwartz, k., and Cooper, N. (1961). Arch. intern. Med., $108,400$.

Weinberger, H. W., Ropes, M. W., Kulka, J. P., and Bauer, W. (1962). Medicine (Baltimore), 41, 35.

Wilkinson, M., and Jones, B. k. (1962). Ann. rheum. Dis., 21. 51.

Evaluation de la biopsie par ponction de la membrane synoviale

\section{RÉSUMÉ}

On analise les résultats de 69 biopsies par ponction réussie de 60 genoux arthritiques.

On discute la valeur de ce procédé et les difficultés d'interprétation du matériel histologique.

Valoración de la biopsia por punción de la membrana sinovial

Sumario

Se analizaron los resultados de 69 logradas punciones biópsicas procedentes de 60 rodillas artríticas.

Se discute el valor del procedimiento y las dificultades de interpretación del material histológico. 